estimated from one-shot measurement than from step response. For one-shot measurement a short adsorption bed is usually preferable. It is interesting that a solid-gas adsorption bed as short as five particle layers may successfully be used in estimating the adsorption equilibrium constant provided that inert beds are installed between test bed and concentration detecting filament. A short adsorption bed can be effectively employed, particularly when the system has a large adsorption equilibrium constant and a long time is required to obtain response signal in a conventional long bed.

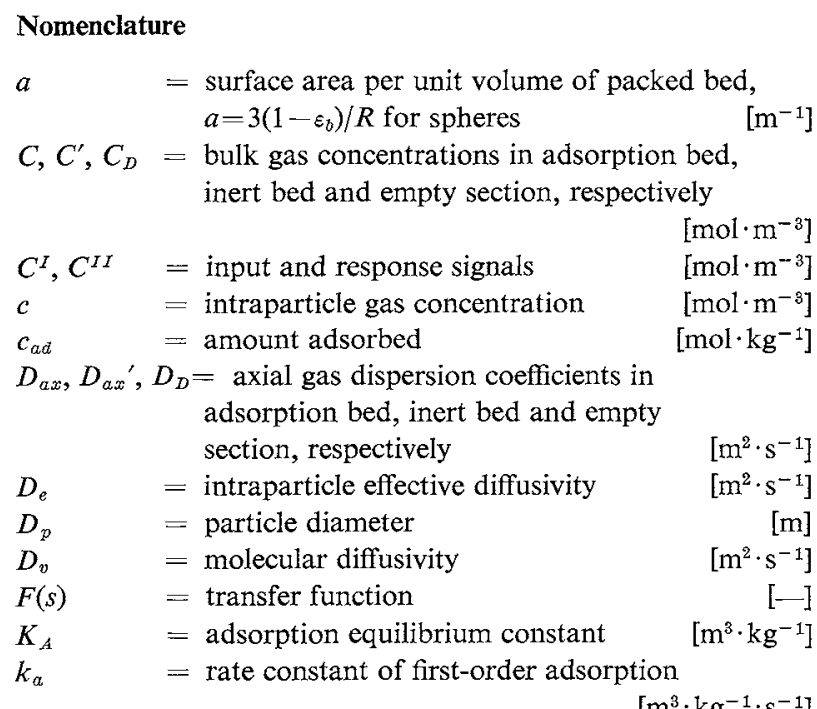

$$
\begin{aligned}
& k_{f} \quad=\text { particle-to-gas mass transfer coefficient } \\
& \begin{array}{l}
L \quad=\text { adsorption bed length } \\
L^{\prime}, L^{\prime \prime}, L_{D}=\text { lengths, refer to Fig. } 2
\end{array} \\
& R \quad=\text { particle radius } \\
& =D_{p} U \varepsilon_{b} \rho_{F} / \mu \text {, Reynolds number } \quad \text { [-] } \\
& \text { Sc } \quad=\mu /\left(\rho_{F} D_{v}\right) \text {, Schmidt number } \quad[-] \\
& \text { Sh } \quad=k_{f} D_{p} / D_{v} \text {, Sherwood number [-] } \\
& U, U^{\prime} \quad=\text { interstitial gas velosities in adsorption } \\
& \text { bed and inert bed, respectively }\left[\mathrm{m} \cdot \mathrm{s}^{-1}\right] \\
& u \quad=\text { gas velocity in empty column } \quad\left[\mathrm{m} \cdot \mathrm{s}^{-1}\right] \\
& \varepsilon \quad=\text { root-mean-square-error, defined by Eq. (13) } \\
& \varepsilon_{b}, \varepsilon_{b}^{\prime}=\text { void fractions in adsorption bed and } \\
& \text { inert bed, respectively } \\
& \varepsilon_{p} \quad=\text { intraparticle void fraction } \\
& =\text { gas viscosity } \\
& =\text { gas density } \\
& =\text { particle density }
\end{aligned}
$$

\section{Literature Cited}

1) Gangwal, S. K., R. R. Hudgins, A. W. Bryson and P. L. Silveston: Can. J. Chem. Eng., 49, 113 (1971).

2) Kaguei, S., K. Matsumoto and N. Wakao: Chem. Eng. Sci., 35, 1809 (1980)

3) Lee, D. I., S. Kaguei and N. Wakao: J. Chem. Eng. Japan, 14, 161 (1981).

4) Schneider, P. and J. M. Smith: AIChE J., 14, 762 (1968).

5) Wakao, N. and T. Funazkri: Chem. Eng. Sci., 33, 1375 (1978).

6) Wakao, N., S. Kaguei and J. M. Smith: Ind. Eng. Chem., Fundam., 19, 363 (1980).

\title{
THE REACTION ORDER OF SOLID CONCENTRATION FOR VOLUME REACTION MODEL
}

\author{
Noboru SAKAI, TADASHI CHIDA AND TEIRIKI TADAKI \\ Department of Mining and Mineral Engineering, Tohoku University, Sendai 980
}

\section{A fluid-solid reaction such as}

$$
\mathrm{A} \text { (fluid) }+\mathrm{S} \text { (solid) }=\text { Products }
$$

has often been analyzed kinetically by the volume reaction model ${ }^{1,3-5}$. In this model, the disappearance rate of component $\mathrm{A}$ per unit volume of bulk solid $\left(-r_{A}\right)$, is assumed to be a function of the fluid and solid concentrations,

$$
\left(-r_{A}\right)=k f\left(C_{A}, C_{S}\right)
$$

Received September 11, 1981. Correspondence concerning this article should be addressed to N. Sakai. T. Tadaki is at Dept. of Chem. Eng
The function $f\left(C_{A}, C_{S}\right)$, however, could not be represented theoretically. Thus the rate has been described approximately by the following form:

$$
\left(-r_{A}\right)=k C_{A} C_{S}^{m}
$$

Chida and Tadaki $^{2}$ reported recently that the solid concentration dependence of the rate can be expressed in the form of Eq. (3), on the basis of a structural model in which a solid pellet is composed of a large number of fine spherical particles. However, the case where $m$ is less than $2 / 3$ could not be explained 


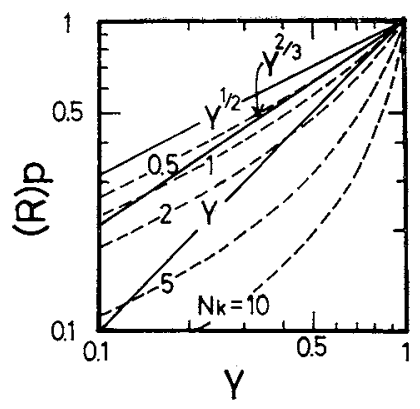

Fig. 1 Relations between $Y$ and $(R)_{p}$ (cylindrical particle)

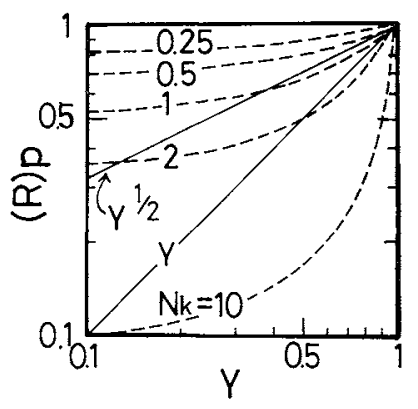

Fig. 2 Relations between $Y$ and $(R)_{p}$ (slab-like particle)

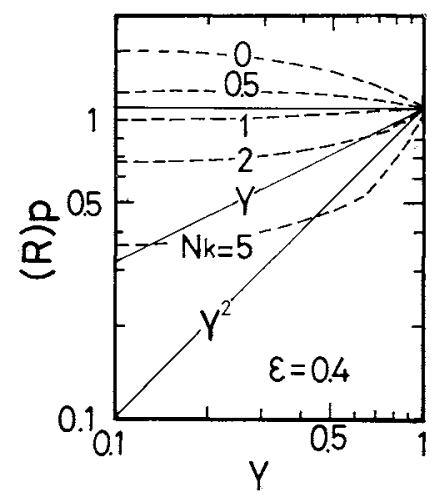

Fig. 3 Relations between $Y$ and $(R)_{p}$ (pore model)

by this consideration.

This paper shows that $m$ can take a value less than $2 / 3$, and even a negative value under extreme conditions, on the basis of other structural models different from that mentioned above.

\section{Assembly Models of Non-Spherical Particles}

\section{1 Cylindrical particle}

Consider that a porous spherical pellet is composed of a large number of fine, dense cylindrical particles whose radii are sufficiently small compared to their length, and that reaction (1) takes place in the particles. It is assumed that the unreacted core shrinking model is applied to the kinetic analysis of the particles and that the disappearance rate of fluid component $\mathrm{A}$ per unit reaction area is given as

$$
\left(-r_{A}\right)^{\prime}=k^{\prime} C_{A}
$$

Then the overall reaction rate of a particle, $\left(-r_{A}\right)_{p}$, is given in the following form based on the analysis in the previous paper ${ }^{2}$ :

$$
\left(-r_{A}\right)_{p}=2 \pi r_{c} l\left(-r_{A}\right)_{r=r_{c}}^{\prime}
$$

And the dimensionless overall reaction rate, $(R)_{p}=$ $\left(-r_{A}\right)_{p} / 2 \pi l r_{0} k^{\prime} C_{A b}$, is represented as follows.

$$
(R)_{p}=\frac{\sqrt{Y}}{1-N_{k} \sqrt{ } Y} \ln \sqrt{ } Y
$$

where $N_{k}=k^{\prime} r_{0} / D e, Y=C_{s} / C_{a 0}$ and $D e$ is the effective diffusivity in the product layer of the particle.

On the other hand, the volume reaction model with Eq. (3) gives the following equation ${ }^{2}$.

$$
(R)_{p}=Y^{m}
$$

Therefore, if Eq. (6) is approximated by Eq. (7), the value of $m$ can be estimated.

Figure 1 shows the relation between $(R)_{p}$ and $Y$ of Eq. (6). If $N_{k}<0.5,(R)_{p}$ is approximated by the function $Y^{1 / 2}$, and if $N_{k}$ is in the range from 0.5 to 1 , $(R)_{p}=Y^{2 / 3}$. When $N_{k}$ is equal to zero Eq. (6) coincides with $Y^{1 / 2}$, and the value of $m$ estimated is always larger than $1 / 2$ in this case.

\section{2 Slab-like particle}

Consider that a porous spherical pellet is composed of a large number of fine, dense slabs whose thickness is sufficiently thin. The other steps in the analysis are the same as those for the previous model.

Then $(R)_{p}=\left(-r_{A}\right)_{p} / 2 A_{s} k^{\prime} C_{A b}$ is given as

$$
(R)_{p}=\frac{1}{1+N_{k}(1-\bar{Y})}
$$

Figure 2 shows the relation between $(R)_{p}$ and $Y$. If $N_{k}<0.5,(R)_{p}$ may be approximated by a constant $Y^{0}=1$. However, if $N_{k}<0.5$ it is difficult for $(R)_{p}$ to be approximated with a function in the form of $Y^{m}$.

\section{Pore Model}

Porous materials can be considered to be network systems composed of numerous cylindrical pores. This consideration means that fluid-solid reaction takes place on the pore wall at the beginning, and then proceeds into the solid centrifugally.

Now assume that all pores in the pellet are of uniform size, and that the solid phase is formed concentrically around the pore from $r_{0}$ to $r_{1}$.

Then the outer radius of the space $r_{1}$ is given by the following equation.

$$
r_{1}=r_{0} \sqrt{ } 1 / \bar{\varepsilon}
$$

The diffusion equation in the product layer $\left(r=r_{0} \sim r_{c}\right)$ is written as

$$
\begin{aligned}
& d(R(d Z / d R)) / d R=0 \\
& \text { B.C. } \quad Z=1 \text { at } R=1 \\
& \quad d Z / d R=N_{k} Z \text { at } R=R_{c}
\end{aligned}
$$


where $R=r / r_{0}, R_{c}=r_{c} / r_{0}$ and $Z=C_{A} / C_{A b}$

The solution of Eq. (10) with the boundary conditions (11) and (12) is

$$
Z=\frac{1+N_{k} R_{c} \ln \left(R_{c} / R\right)}{1+N_{k} R_{c} \ln \left(R_{c}\right)}
$$

In this equation $R_{c}$ is given as follows.

$$
R_{c}=\sqrt{(1-(1-\varepsilon) Y) / \varepsilon}
$$

Then the dimensionless overall reaction rate, $(R)_{p}=$ $\left(-r_{A}\right)_{p} / 2 \pi l^{\prime} r_{0} k^{\prime} C_{A b}$, can be written as

$$
(R)_{p}=R_{c} /\left(1+N_{k} R_{c} \ln \left(R_{c}\right)\right)
$$

Figure 3 shows the relation between $(R)_{p}$ and $Y$ in the case of $\varepsilon=0.4$. If $0.5<N_{k}<1,(R)_{p}$ may be approximated by a constant $Y^{0}=1$, but it is difficult in the case of other values of $N_{k}$ for $(R)_{p}$ to be approximated with a function in the form of $Y^{m}$. One should note that if $N_{k}<0.8,(R)_{p}$ is larger than unity. This means that $m$ is negative; that is, the reaction is accelerated with decrease of solid reactant. This phenomenon may be one of the explanations for sigmoidal conversion-time curves in fluid-solid reactions.

\section{Conclusion}

It is found on the basis of structural models and the pore model that the reaction order of solid concentration for the volume reaction model may take a positive value, say, $0,1 / 2$ or $2 / 3$, and under extreme conditions a negative value.

\section{Nomenclature}

$A_{S} \quad=$ reaction surface area of solid

$\left[\mathrm{m}^{2}\right]$

$$
\begin{aligned}
& C_{A}, C_{A 0}=\text { concentration of } \mathrm{A} \text { in particle and bulk } \\
& C_{S}, C_{S O}=\left[\mathrm{mol} / \mathrm{m}^{3}\right] \\
& C_{S}, C_{S 0}=\text { concentration and initial concentration } \\
& \text { of } \mathrm{S} \quad\left[\mathrm{mol} / \mathrm{m}^{3}\right] \\
& D_{e} \quad=\text { effective diffusivity }\left[\mathrm{m}^{2} / \mathrm{s}\right] \\
& k \quad=\text { rate constant defined by Eq. (3) } \quad\left[\left(\mathrm{m}^{3} / \mathrm{mol}\right)^{\mathrm{m}} / \mathrm{s}\right] \\
& k^{\prime} \quad=\text { rate constant defined by Eq. (4) } \quad[\mathrm{m} / \mathrm{s}] \\
& l \quad=\text { length of a cylindrical particle [m] } \\
& l^{\prime} \quad=\text { length of a cylindrical pore } \quad[\mathrm{m}] \\
& m \quad=\text { reaction order } \quad[-] \\
& N_{k i} \quad=k^{\prime} r_{0} / D_{e} \quad \text { [-] } \\
& (R)_{p} \quad=\text { dimensionless overall reaction rate } \\
& r \quad=\text { radius coordinate [m] } \\
& r_{0} \quad=\text { radius of cylindrical praticle or pore } \quad[\mathrm{m}] \\
& r_{1} \quad=\text { imaginary outer radius of solid defined } \\
& \text { by Eq. (9) } \\
& \left(-r_{A}\right) \quad=\text { disappearance rate of A per unit volume } \\
& \text { of bulk solid }\left[\mathrm{mol} / \mathrm{m}^{3} \cdot \mathrm{s}\right] \\
& \left(-r_{A}\right)^{\prime}=\text { diasppearance rate of A per unit reaction } \\
& \text { area } \\
& \left(-r_{A}\right)_{p}=\text { reaction rate of a particle }[\mathrm{mol} / \mathrm{s}] \\
& r_{c} \quad=\text { radius of reaction interface [m] } \\
& Y \quad=\text { dimensionless solid concentration } \quad[-] \\
& Z \quad=\text { dimensionless fluid concentration } \quad[-] \\
& \varepsilon \quad=\text { volume fraction of macropore } \quad[-]
\end{aligned}
$$

\section{Literature Cited}

1) Borghi, M. D., J. C. Dann and K. B. Bischoff: Chem. Eng. Sci., 31, 1065 (1976).

2) Chida, T. and T. Tadaki: J. Chem. Eng. Japan. 13. 410 (1980).

3) Dudukovic, M. P. and H. S. Lamba: Chem. Eng. Sci. 33, 471 (1978).

4) Fan, L. S., K. Miyanami and L. T. Fan: Chem. Eng. J., 13, 13 (1977).

5) Lamaba, H. S. and M. P. Dudukovic: ibid., 16, 117 (1978).

\title{
CRITERION FOR SIMULTANEOUS INTERPHASE TEMPERATURE AND CONCENTRATION GRADIENTS
}

\author{
T. CHAKRABARTY, R. R. HUDGINS AND P. L. SILVESTON \\ Department of Chemical Engineering, University of Waterloo, Waterloo, Ontario, Canada
}

A number of theoretical criteria for avoiding significant interphase temperature and concentration gradients have been developed for each gradient independently. Butt and Weekman ${ }^{1 /}$ summarized criteria published before 1974. Since both thermal concentration gradients may occur simultaneously

Received September 24, 1981. Correspondence concerning this article should be addressed to P. L. Silveston. T. Chakrabarty is now with Esso Resources Canada L.td., Research Department, Calgary, Alberta. near the surface of a catalyst pellet, a combined criterion would be more appropriate. The reason: the simultaneous existence of temperature and concentration gradients may have either a compensating or an amplifying effect on the global rate, For example, the effect of a temperature gradient in an exothermic reaction is to increase the global rate. Consequently, in an exothermic, positive-order reaction (e.g. $\mathrm{CO}$ oxidation on $\mathrm{V}_{2} \mathrm{O}_{5}$ ), the two gradients may 